\title{
Recognition of the role of nature in the formation of fractal architecture
}

DOI 10.1515/otmcj-2016-0020

Received January 15, 2017; accepted May 20, 2017

Abstract: After a long period of one-way consumerist attitude toward nature, there have been some alternate perspectives on the systemic relationship between humans and nature, which have been again brought up during the past few decades. Since the late twentieth century, fractal architecture has been one of the most important themes discussed in architecture and it is based on the chaos and complexity theories. Critics often criticize this architecture due to its lack of architectural values, practicality, and attention to economy, culture, and history. The current study aims to clarify the scientific theories that are the theoretical foundations of this approach in contemporary architecture. By categorizing the practical examples of this architectural approach, they are analyzed in terms of their relationship with nature using the logical reasoning method to achieve a favorable architecture. In fact, the gap between this architecture and nature's behavior is shown.

Keywords: chaos theory, fractal architecture, form, geometry, nature

\section{Introduction}

After the fall of man from the original abode, i.e., the supernatural world, to the natural world, he had to live within and with nature, in spite of attachment to his sublime memories. He gradually realized that not all natural conditions were appropriate, and to ensure his comfort, he had to manipulate nature and create a synthetic environment alongside with nature. As a result, different environments were created by humans in the heart of nature, sometimes different from each other in terms of climate and geography and sometimes they differed based on the creators' intellectual infrastructure and their definition of nature and its relationship with humans.

There has been a long period of one-way consumerist attitude toward nature rooted in a nonsystemic relationship between nature and humans, as well as lack of understanding of man's real needs in the modern era, called "conflict with nature" when humans have dominated the nature; this can be traced clearly in contemporary architecture. There were some viewpoints on the systemic relationship between humans and nature called "naturalistic" perspectives, which have been again brought up during the past few decades. In the emerging attitudes in architecture during recent years, the general view has changed from "nature seeking" to "nature studying" to resolve the several crises arising from the failed relationship between humans and nature.

Since the late twentieth century, one of the most important themes in architecture has been fractal architecture, rooted in the chaos and complexity theories. Some architectural theorists categorize it as the "new organic architecture" discussed in the organics theory (Pearson 2001). A multifaceted analysis of fractal architecture is now available from difference sources. According to critics, during interdisciplinary communications, a part of this theory has been always interpreted from the perspective of the other disciplines and that is why some of its parts have been ignored or eliminated. Critics often criticize fractal architecture due to its lack of architectural values, practicality, and attention to economy, culture, and history.

The present study aimed to discuss the contradictory perspectives of architects with respect to nature and scientific theories, i.e., the gap between architecture and naturalism.

\section{Methodology}

*Corresponding author: Seyedeh Somayeh Mirmoradi, Assistant Professor, Architecture Department, School of Civil Engineering, Babol Noshirvani University of Technology, Babol, Iran

mirmoradi81@gmail.com ors.mirmoradi@nit.ac.ir
The main areas of the research include fractal geometry, chaos theory, complexity theory, and fractal architecture. 
The research background, i.e., analyzing the different existing themes of fractal architecture, introducing relevant theories, clarifying the manner in which fractal architecture can use these theories by categorizing the existing examples and analyzing how to use these theories, assessing the closeness to solutions and practical examples, and comparing architectural examples and natural behaviors, shapes the body of this research. Logical reasoning has been used in the analytical stage of the research.

\section{Research background}

Review and analysis of fractal architecture from various aspects are available from different sources. According to critics, during interdisciplinary communications, a part of the theory has been always interpreted from the perspective of other disciplines and that is why some of its parts have been ignored or eliminated. Critics often criticize this type of architecture due to its lack of architectural values, practicality, and attention to economy, culture, and history. For instance, Martin Pawley (1994), in an article published in The Observer mentioned that architecture based on complexity is folly in which a lot of money is spent on zigzag buildings (Pawley 1994). In response to this objection, Jencks stated that Le Corbusier, in his book entitled Toward a New Architecture, referred to the fact that some eyes were deprived of looking and now Pawley cannot see the beauty of new forms in nature (Jencks 2004, pp. 140-141). Likewise, we can refer to Michael Sorkin (1991), who considers chaos as an unfamiliar term, especially in the case of architecture. He believed that there is no reason to use it in architecture (Sorkin 1991, pp. 346347, as cited in Ostwald 2001). According to Aaron Betsky, saving Eisenman's architecture from annihilation against the fractal geometry is regarded as one of the advantages of Euclidean geometry, although Eisenman claimed that the biology center is the combination of Euclidean, non-Euclidean and fractal geometries (Aaron Betsky 1990, p.148, as cited in Ostwald 2001). The repeated use of other classifications, including "Fractalism", "Complexitism", "Complexity Architecture" and "Non-linear Architecture", have led scholars such as Yannick Joye to argue that "a systematic, encompassing, scholarly treatment of the use and presence of this geometrical language in architecture is missing” (Joye 2011, p. 814). Paul Shepheard also addressed Eisenman and Libeskind as wizards who design with closed eyes and considered their works as being against nature, scrawling and doing whatever they wish (Paul Shepheard 1994, p.15, as cited in Ostwald 2001).
Christopher Langford also mentioned that fractal is only a fashionable terminology (Christophe Langhof 1994, p.41, as cited in Ostwald 2001). One of the essential problems when considering such cross-disciplinary connections is that many different types of relationships are possible among seemingly diverse fields. This problem is exacerbated when architecture is considered, because design serves a wide range of functions, from the physical to the social and the symbolic (Ostwald and Williams 2015).

There are other examples of criticisms to fractal architecture in which the architects analyze the architecture in terms of efficacy, performance, and economy.

However, such past attempts to compare nature and architecture using fractal analysis have not been entirely convincing. The disparity of the methodological variables used in each approach has been cited as the primary reason that the fractal data derived from nature cannot be easily compared with equivalent architectural data. In particular, representational approaches to the analytical images are disparate, inconsistent, and uncategorized (Vaughan and Ostwald 2017: 156). The current study aimed to discuss the contradictory viewpoints of architects with nature and scientific theory, i.e., the gap between architecture and naturalism.

\section{Introduction to relevant scientific theories}

The chaos theory was first proposed by Edward Lorentz in 1963, which aimed to implement qualitative research on natural behaviors. Here, the main idea is the recreation and modeling of natural behavior, which is influenced by fractal architecture. According to the theorists, this theory is supplementary to classical Euclidean geometry. Fractal geometry can be used in natural behavior modeling and describing natural shapes, such as snow crystals, leaves, natural wrinkles, and "amorphous" forms in Euclidean geometry.

Mandelbrot, who is among the first theorists and inventors of fractal geometry, indicated that clouds are not like spheres, mountains are not cone-shaped, and lightning does not strike in a straight line (Mandelbrot 1992: 53). He believes that a new geometry reflecting a rough globe, which is noncircular, uneven, and nonlinear, with forms such as wrinkles, curves, and bends, is necessary to describe nature. This theorist adopted the name from a Latin word "fractus", meaning a stone crushed in an irregular way (Mandelbrot 1992: 52). He regarded geometry as a means of describing a part of the complex nature. 
In fact, he was looking for a way to detect the hidden forces shaping natural forms and to recreate these forms using those forces (Mandelbrot 1992: 52) (Figure 1).

To present his geometry, Mandelbrot (1992) described the characteristics of fractal natural forms, such as the disorder of fractal forms in the conventional sense. Despite the lack of standard order, their level of irregularities was equal in every scale, i.e., they are organized in another way. Furthermore, as fractal objects are self-similar, they are observed to be identical from far and near. When we approach a fractal object, we can see that objects that looked like amorphous seeds from a distance turn out to be clear objects (Mandelbrot 1992: 52). Moreover, these shapes are not straight-edged forms but with jagged edges. To describe this feature, Mandelbrot cited an example in which a few people measured the coastline but, due to the jagged edge on an infinite scale, they came up with different values, i.e., the smaller the scale of the map, the finer the details would be. Therefore, the edge was jagged (Mandelbrot 1982: 39).

The chaos theory is defined in different ways. Ian Stewart, the mathematician in this field of study, believed that chaos is an ability of simple patterns without internal random characteristics that leads to irregular behaviors (Sardar 2001: 11). In other words, chaos is a qualitative study of nonperiodic unstable behaviors in nonlinear, determinable, and dynamic behaviors (Sardar 2001: 11).
Determinable system is predictable and stable. Nonperiodic unstable behavior can be regarded as a behavior that never repeats itself and that can be influenced by any system failure. Such behavior makes accurate prediction impossible and then causes the values seem to be random. In fact, the analysis of these patterns and behaviors is known today as the "chaos theory". One of the key features of these systems is that some behaviors never occur, even those that are expected. Those behaviors that occur belong to a particular area called "attractors", which are used to illustrate the situation of a system at any point in the phase space. In phase space, the full status of a dynamic system is accumulated at a certain point representing the system behavior at that moment.

When drawing phase space with these aforementioned specifications, we come across shapes and attractors called "strange attractors". In fact, phase space from strange attractors is one of the behavioral traits of chaotic systems. The most well-known strange attractor is called "Lorenz" since it was first discovered by Lorenz (Sardar 2001: 54) (Figure 2).

Self-organizing is another behavioral trait of systems with chaotic behaviors. Ilya Prigogine is regarded as another pioneer of the chaos theory, who first proposed the concepts of self-organizing and dissipative systems. He stated that when a system without equilibrium goes into a chaotic period, it naturally achieves a different level of order. This process is called "self-organizing".

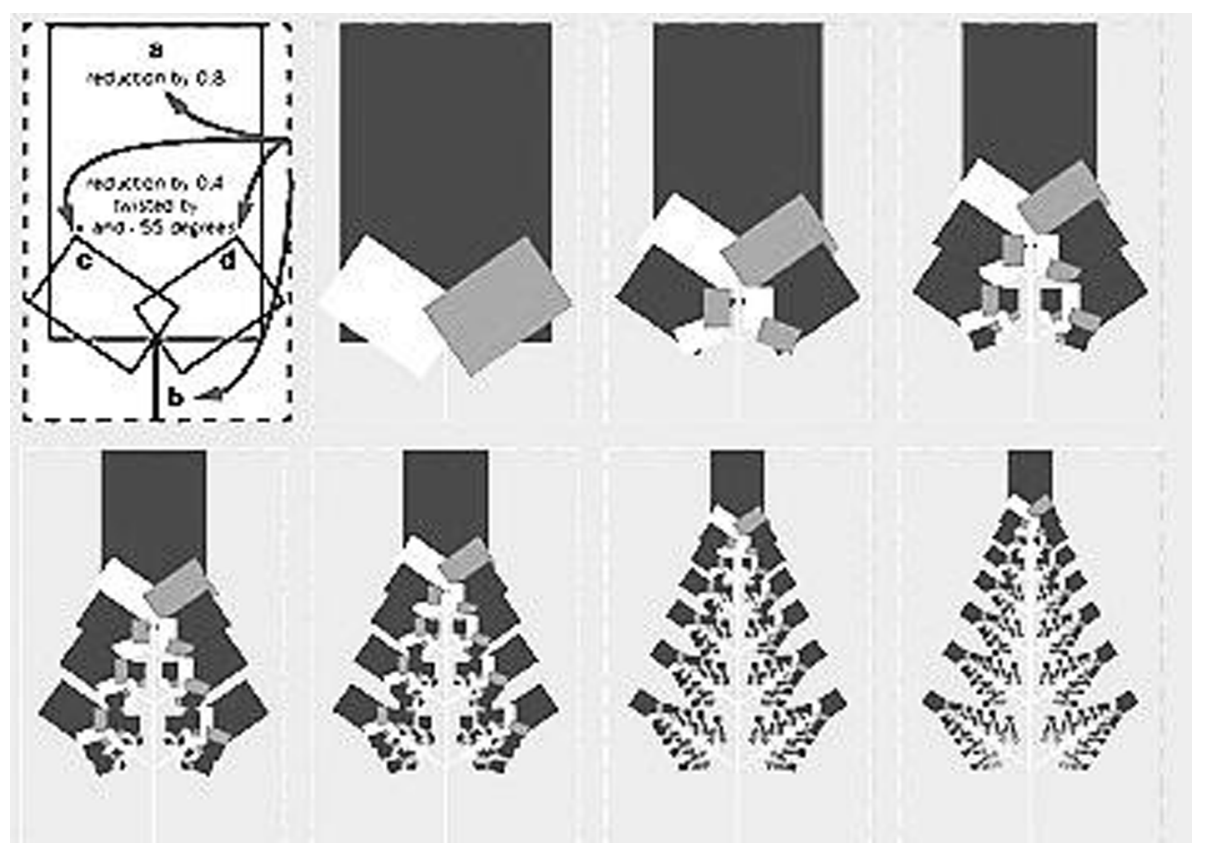

Fig. 1: Types of fractals: fractals generated with repetitive operations. According to Lorenz (2003), these fractals are based on the relationship between mathematics and nature, in which the basic form is duplicating due to change in scale and rotation. 


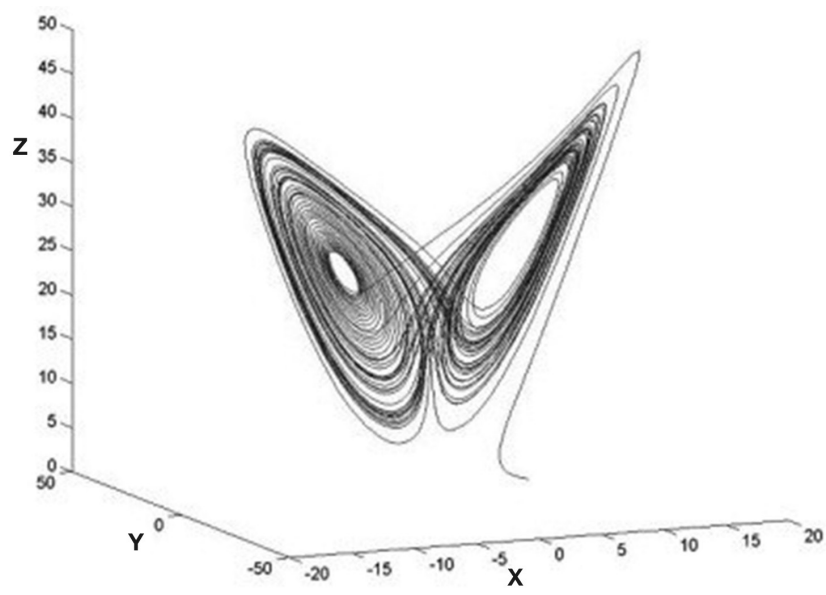

Fig. 2: Strange attractor. An example of strange attractors, known as the "butterfly effect", was given by Lorenz (2003).

Accordingly, a book entitled Order out of Chaos has been published by Prigogine in 1984. Prigogine defined self-organizing as a phenomenon in which the system organizes its internal structure independent of external causes. Such self-organizing systems show other chaotic characteristics such as nonlinear trait, feedback, fractal structures, and sensitive dependence on initial conditions (butterfly effect) (Sardar 2001).

However, in the 1980s, the phenomenon of "edge of chaos" drew the attention of most of the scholars in different scientific fields and represented a new theory called "complexity theory". In fact, nonlinear dynamic systems being studied by the chaos theory were complex systems. This means that there are a large number of independent variables interacting with each other in various ways. These complex systems have the potential to balance between order and chaos, called "edge of chaos", in which the system is suspended in-between stability and complete fall. At this moment, the selforganizing process occurs (Sardar 2001: 84). Indeed, the richness and diverse actions of a large number of independent variables enable complex systems to selforganize themselves.

Adaptability is considered another feature of complex systems. The complex systems are not passive but they react actively to turn each event into their benefit. Similar to different species that adapt themselves to environmental changes easily, complex systems show clearly the mutual relationship between objects, such as trees and weather, people and environment, communities with each other, and so on (Sardar 2001, p. 86). In fact, chaos and complexity theories are considered the means for better understanding of phenomena, particularly the natural phenomena.

\section{Examples of fractal architecture}

In the late twentieth century, a new architectural approach emerged from the relationship between architecture and scientific theories, such as chaos theory, fractal geometry, and complexity theory. According to the architecture analysts, substantial parts of each theory had been ignored when these theories were applied. The features and characteristics of these theories are discussed as follows.

1. Using one of the properties of fractal shapes, such as self-similarity, scaling, and fractal dimensions

- House 11a. Peter Eisenman is one of the most important designers who have used the properties of fractal shapes. According to the designer, the building has been scaled in itself repetitively, which refers to self-similarity of fractal shapes (Eisenman 1988: 70). Furthermore, the designer has used a set of L-shaped forms with various scales. According to Eisenman, it has the shape between square and rectangular, presenting the in-between shape of fractals. The presence of an object in another object shows the main form referring to the detail-to-general similar characteristics in fractal objects (Figure 3).

- Then, Eisenman implemented the Canarjio plan using a series of self-similar objects of different scales. In the plan, he selected these objects from among a large number of objects, which can only be used in a desolate house.

- Joe Price Bruce Goff's work is another example that Charles Jencks considers as one of the best fractal architectures in his book entitled The Architecture of the Jumping Universe. Regarding this building, Jencks stated that Goff 's design is an imitation of natural patterns and repetition of an idea in different scales, such as a flower in different shapes, i.e., triangles, hexagons, and pentagons, 60-degree angles, divided and squared. In fact, Goff invented fractal architecture. He also argued that self-similarity in fractals is not the same as self-identity. Self-similarity influenced by natural fractals is considered positive, although self-identity has resulted in tedium in modernism (Jencks 2004: 87) (Figure 4).

Galinsky School designed by Zvy Hecker is another example of repeating a form at different scales with rotation and resizing of the basic shape. Here, the basic shape is a triangle (Figure 5). 
2. Using a natural geometric shape representing strange attractors

In this method, the designer proposes a general form derived from natural ones as a general form and then creates the details accordingly. As mentioned earlier,

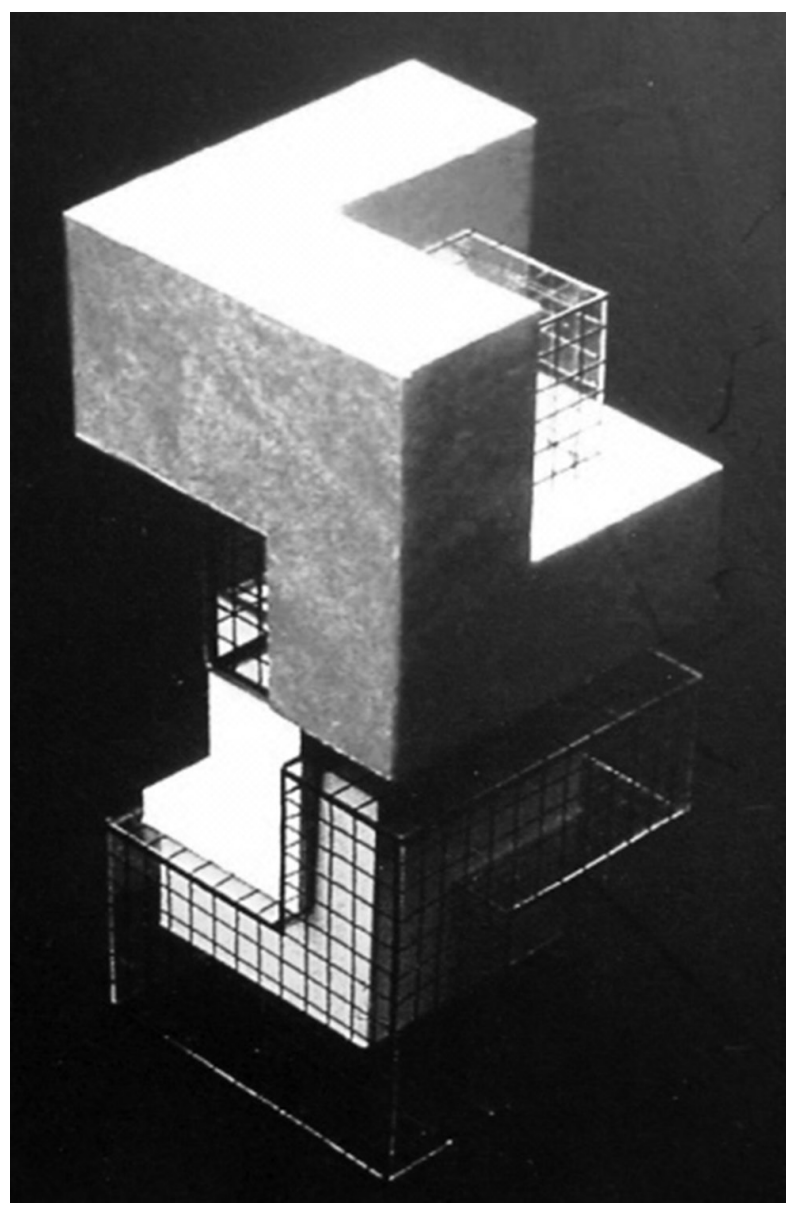

Fig. 3: Self-similarity of fractal shapes in a general form of architecture - repeating a form at different scales (House 11a, designed by Peter Eisenman). the behaviors of strange attractors must occur within their own area. Jencks believed that Bruce Goff used the spiral form in Bavinger House based on the strange attractor principle in natural behaviors and the path forming the whole plan is regarded as the strange attractor.

He claimed that in Bavinger House, the rooms are like attractor points in a spiral path rotating around the central ring, acting as a strange attractor. The spiral trajectory attracts movement and visual guidance to itself, acting differently in each and every up and down turn (Jencks 2004: 91) (Figure 6).

\section{Using the formation process of fractal shapes}

Mathematical fractals are examples of fractals used for modeling the natural shapes in which the basic shapes are repeated and duplicated by resizing and rotating. First, similar to mathematical fractals, a basic shape is selected and duplicated by resizing and rotating. Then, in the next stage, the final plan is designed considering the project limitations. In fact, the components altogether create an unpredictable whole. The determining factor of a whole is its components, although the whole is totally unpredictable to us.

As shown in Figure 7, Opera Cardiff by Greg Lin and Claire is an example of this design method.

\section{Using the complexity and chaos theories}

According to the second law of thermodynamics, entropy and disorder are steadily increasing in the world. However, according to the complexity theory, the natural complex systems are able to self-organize themselves by reaching the chaos edge when there are various independent variables; therefore, they return to order. The systems studied by chaos theory are complex suspended between order and disorder, and

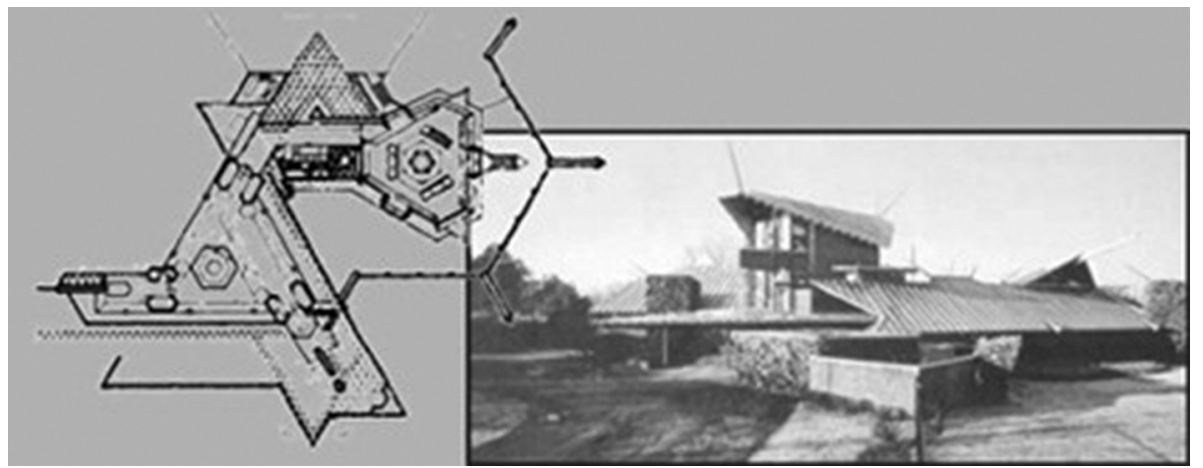

Fig. 4: Joe Price Workshop by Bruce Goff uses the triangle as a basic shape and repeats it at different scales and angles to form a general architectural plan. 
as soon as they reach the edge of chaos, the systems reorganize themselves and restore order out of disorder. The complexity theory is one of the significant theories for architects. Ostwald pointed out that while mathematicians and researchers emphasize geometry

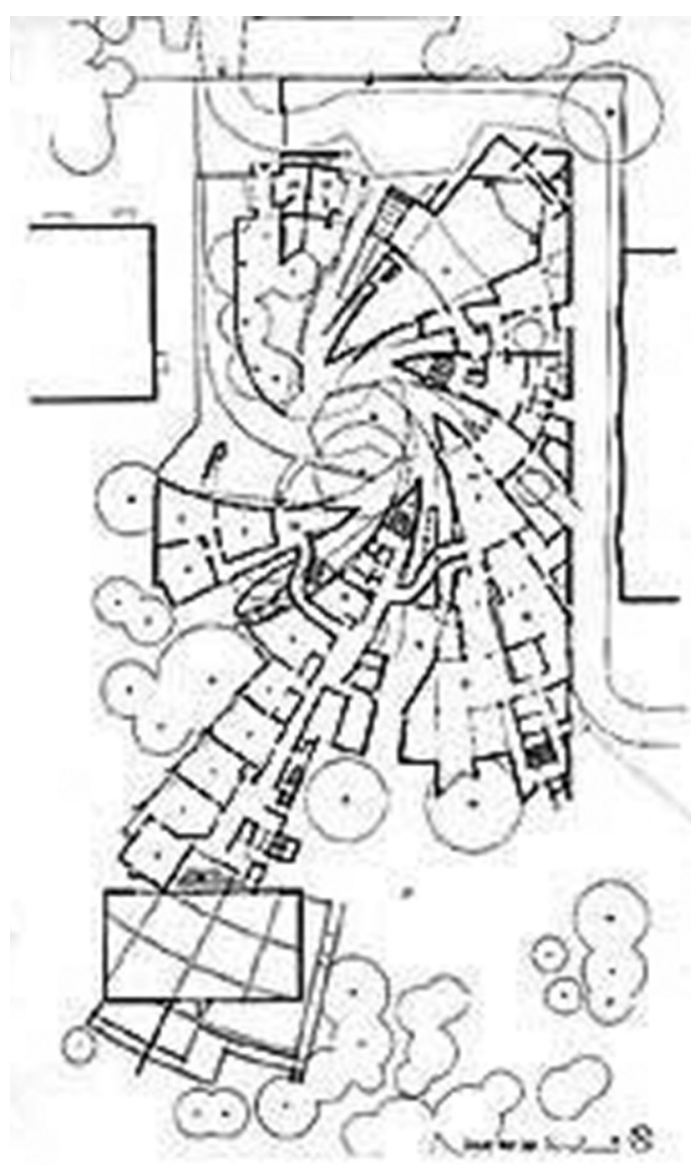

Fig. 5: Galinsky School by Zvy Hecker uses the triangle as a basic shape and repeats it at different scales and angles to form a general architectural plan. for its own sake, architects consider it important due to its relation with the complexity and chaos theories (Ostwald 2001). Concerning this method of architecture, Charles Jencks describes the architect as a god who creates new worlds, however, the one who creates the orders, defectively (Jencks 2004: 118). Then, he calls these defective and unpredictable elements as a lasting solution of architectural works. By creating such a defective work, it seems that Jencks intends to deliver the work to the edge of chaos, i.e., the highest level of complexity, which then is left to the viewers to complete it in their minds and interpret it differently. He considers it as a lasting and dynamic factor over the course of time.

According to Jencks (2004), Frank Gehry’s Guggenheim Museum is an example of a building in accordance with the principles of complexity theory. He believes that since the principles of evolution have tendencies toward more complexity and our plan should be a continuation of the natural world, it should be complex and be diverse so that the system can grow in terms of dimension, energy, and information to achieve complexity. Thus, our architecture is being built like the world today.

The Jewish museum by Daniel Libeskind is another example in which Jencks realized that there are some zigzag forms, failures, and deviations in different ways that direct us to the complexity theory. The exclusive language of this design has a fractal quality based on metaphysical cosmic complexity (Jencks 2004: 117).

\section{Calculating the building façade dimension and scaling the details}

The closer we get to a natural shape, the newer is the information we obtain, leading to a diversity of the natural shapes, apart from their distance. The same is true about

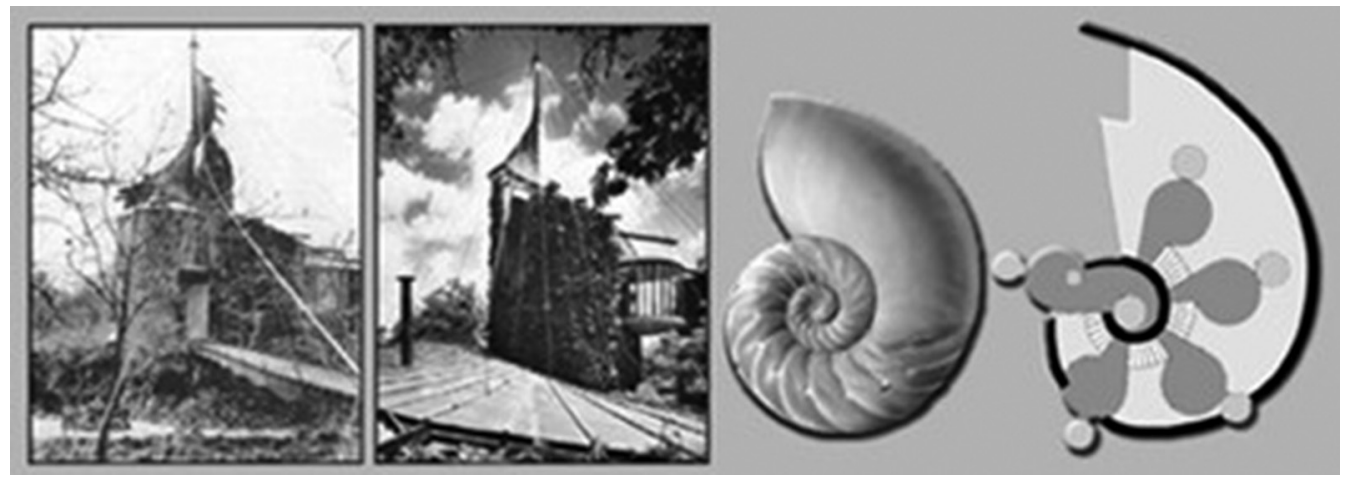

Fig. 6: Bavinger House, by Bruce Goff uses a general shape as strange attractor to show the general idea and organizes the whole plan around it. 

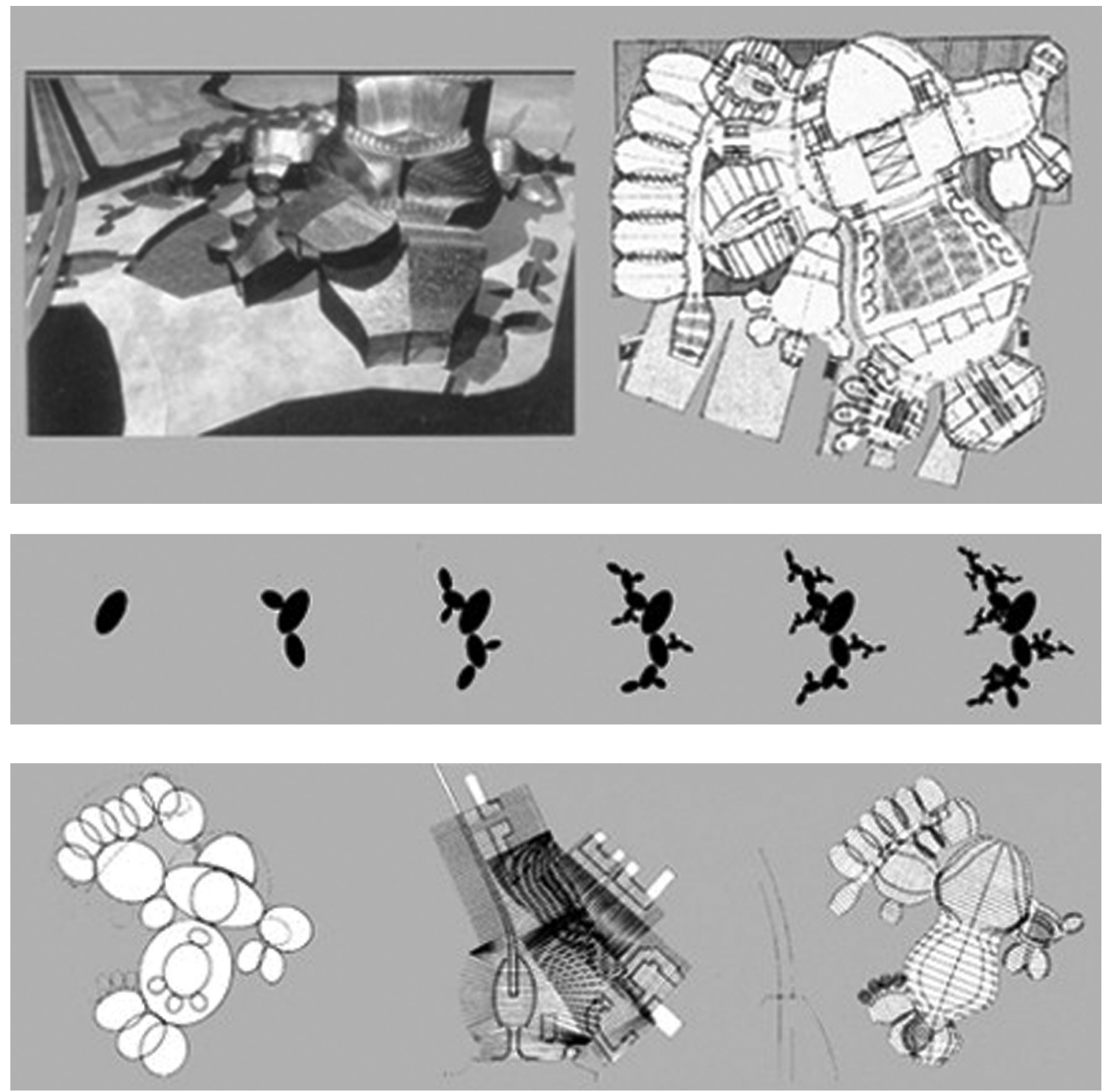

Fig. 7: Opera Cardiff by Greg Lin and Claire (this type uses the formation process of fractal shapes, repeating and duplicating a basic form at different scales and angles as a general plan; Lorenz 2003).

trees (Mandelbrot 1982: 20) (Figure 8). This feature of natural shapes has come into architecture as follows:

Based on the information and memory theory, it would be better to observe order and surprise to some extent. Therefore, as we get closer to the building, there would be always new information for us and we can feel the need to be surprised and to learn new things.

Using the box-counting dimension is a method proposed by Carl Bovill. He examined Ruby Wright House and Glass House of Mies van der Rohe and found that as one gets closer to Ruby's House, there is almost new information as before and the existing lines in the counting box become countable, as there is a reduction in the scales. However, as one gets closer to the glass house and the counting box dimension becomes smaller, the number of lines and information fall constantly (Bovill 1996: 130) (Figures 8 and 9).

\section{Analysis of fractal architecture designs and its relation with nature}

As mentioned earlier, the first designs were the plans in which some properties, such as self-similarity and component similarity to the fractal shapes as a whole, were used. In some natural shapes, these features are formed in one 


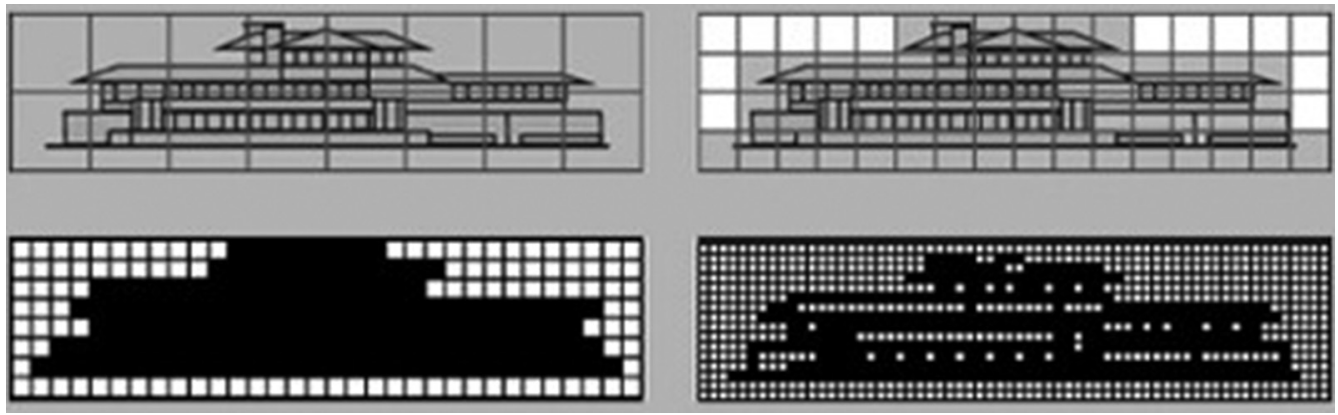

Fig. 8: Ruby Wright House (the box-counting dimension is used to determine whether the scale changes can result in new information or the building information is decreasing while approaching; Lorenz 2003).

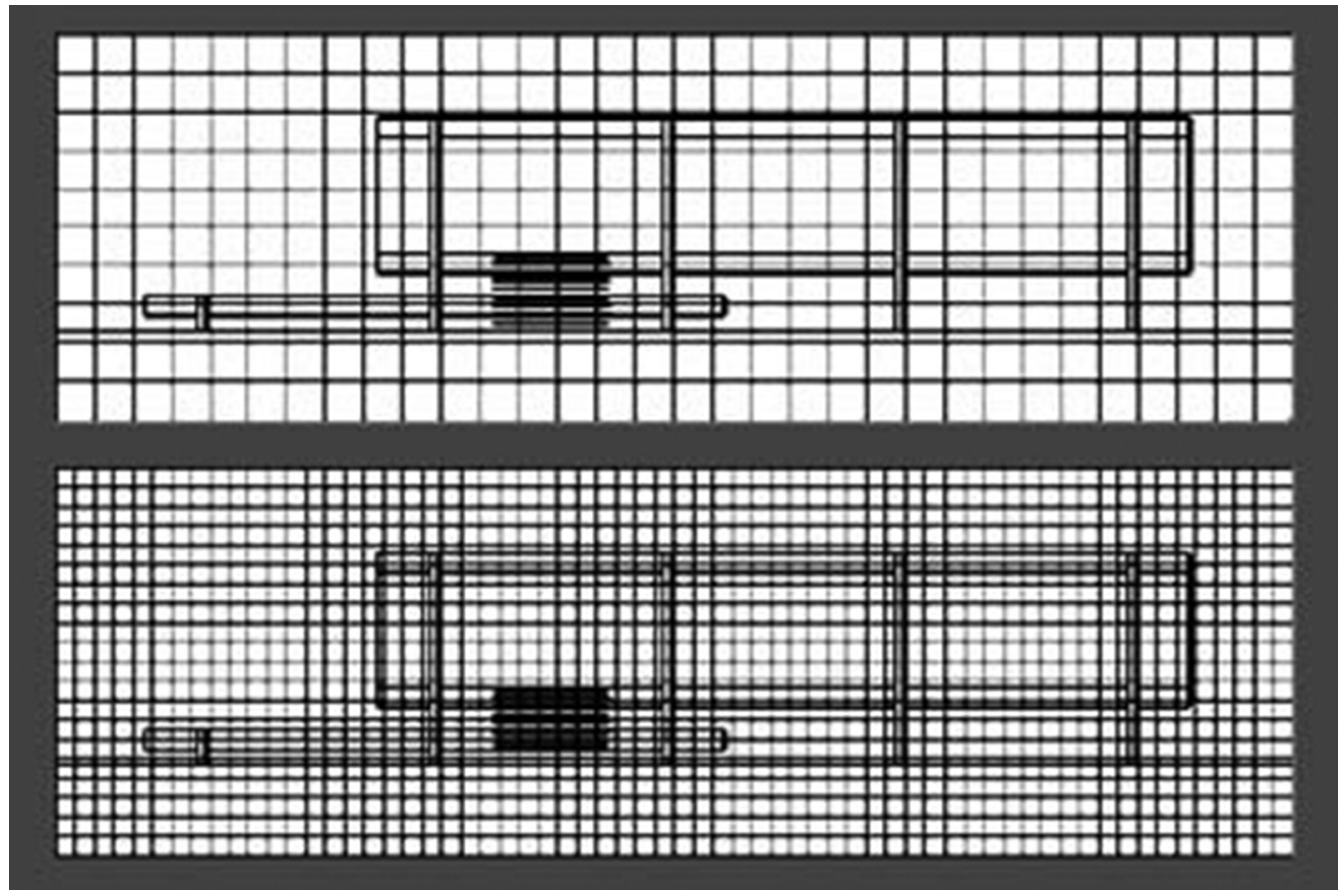

Fig. 9: Glass House of Mies van der Rohe (on getting closer to the building, fewer lines are visible. The information decreases by the changes in the scale; Lorenz 2003).

scale, although these features do not exist in the whole of nature. For instance, the principles that govern the growth of plants such as cauliflower and ferns caused the component similarity due to similar tasks of their different parts since all forms in nature have been created according to existential cause, needs and tasks in the whole of system. This similarity only exists in systems whose different parts do similar tasks. However, the more complex systems such as human bodies, which have several subsystems, are deprived of such geometrical properties. Therefore, this geometry and its properties do not belong to the whole nature but they are in special forms with similar forming forces. As a result, we cannot generalize these mentioned properties in fractal geometry to the whole of nature, which Mandelbrot has referred to, since there are larger systems such as plants, animals, and humans containing various components with organized geometry and we often witness these properties in nonliving and very simple forms in nature. The architecture can be considered similar to larger natural systems in terms of multitude of subsystems and behavioral complexity.

Moreover, in these special shapes in the nature, self-similarity occurs at different scales, not at one scale. What is meant by scaling is that the smaller they become, the closer we get to the shapes, i.e., the size of the components in comparison with the distance to the object. However, in these architecture plans, at a certain scale and distance, the similar components are integrated. 
Furthermore, self-similarity as one of the properties of natural forms has not occurred only by the integration of some self-similar components, but the most essential factor in forming them is the systematic nature of their formation method, by which the components are created by the whole and the whole is created from the components. These forms seem to come together, but naturally, they are not dependent on each other. There is no natural or synthetic reason behind it. They only benefit from a regular trait in nature and the lowest level of order, meaning putting a series of similar forms together. James Harris soundly and correctly rejects Eaton's definition, stating that it "points out the misconception that a repetition of a form ... constitutes a fractal quality. It is not the repetition of the form or motif but the manner in which it is repeated or its structure and nesting characteristics which are important" (Harris 2007: 96). However, the existing order in natural forms has a higher level of order, which has been totally neglected.

The second approach is related to the use of strange attractors, which overshadows the rest of the plan details. In another method, the general plan can be achieved by putting together the components, resizing, rotating, and duplicating them. In other words, either a whole shapes the components in an algebraic form or the components achieve an unpredictable whole in a mathematical and nonfunctional manner.

The important principle in nature is that attractors govern the components, modifying their directions constantly due to the imposed limitations. In other words, the relationship between the whole and its components is not the same as mentioned earlier, but it is a reciprocal move modifying constantly on the way to achieve the whole and, at the same time, the components and the whole control each other. As a result, in these designs, a particular geometry, which is used only due to its similarity to a natural shape and not because of geometrical needs, determines the form and components. The components are integrated to form an unpredictable shape at different sizes and component relationship and there is no control over it. As mentioned earlier, in fact, neither of these methods are based on what occurs in nature and it would be a misinterpretation of the relation between a part and a whole in the nature.

Using the edge of chaos is a creative process of nature in regular shapes under complex conditions, which has been discussed earlier. Nonlinear dynamic systems being studied in the chaos theory are complex systems with many variables that are able to organize the system when it reaches the edge of complexity. In fact, no system in the nature is left before or in the edge of chaos, but whenever it reaches this point, the systems becomes organized and restores order in itself.

This principle might be used in the process of architectural design, claiming that creativity always emerges from a restricted and difficult situation with different variables and alternatives. However, creating the shapes with the highest level of complexity and claiming that these shapes have now reached the edge of chaos similar to what happens in nature is too strange. Here, the chaos and complexity theories are like the means to show and understand the nature's hidden order turned into excuses for lack of order and control. In this regard, Paul Stewart claims that the word "chaos" has been a little bit far from its original place and somewhat degraded. For many people, chaos is a new synonym of "crash". It means one can introduce an unknown system as an example of "chaos" to immediately leave the old and boring areas to reach new and innovative thoughts. Chaos has turned into a metaphor, but mostly a false one (Sardar 2001:171).

Carl Bovill in his book Fractal Geometry in Architecture and Design used the box-counting dimension as a principle of nature design. It is associated with fractal geometry in terms of details scaling in natural fractals. Based on this principle, the size of details in nature has been chosen based on scales, our distance, and the extent to which our visual system is able to see that specific object. In fact, it can be applied in architecture in the form of details in smaller scales or, according to Salingaros, fractals in human scales (Salingaros 2004: 28). It can be helpful in human architectural design if it is not used superficially (when unnecessary similar shapes that are getting smaller constantly are used in the plan). As a matter of fact, this principle criticizes the buildings with smooth walls at human scales, which are nothing but tall and spacious walls built frequently in the modern era (Salingaros 2004: 28)

\section{Discussion and conclusion}

As mentioned earlier, the results from new sciences are aimed at better understanding of nature, being inconsistent with the purposes of these theories. It seems that few parts of these theories have been gleaned and consequently appeared in the plans. Evans maintains that new forms of geometry are always immediately appropriated from mathematics and used by architects regardless of whether or not they are well understood. The danger of this practice is that if the appropriated concept (in this case, fractal geometry) is poorly understood, it may destabilize the theoretical foundations of the design and 
simultaneously undermine the critical intentions of the architect (Ostwald 2010: 127). Applying the sciences in architecture and natural sciences in a reduced and disintegrated manner does not have natural and practical results. In other words, when an activity is done because of its similarity to nature, the results are far from facts and natural principles since the main cause has been totally forgotten. Burckhardt considers wisdom as a key factor to interact with nature. Every work of art should be studied based on its own creation principles and be understood as what it is (Burckhardt 2003: 132). Nevertheless, such a naturalistic view should be respectful since they have understood the importance of nature and the necessity for cooperation. The superficial attitude toward nature has caused many of the works that do not meet the basic requirements of an architectural monument to be criticized by some critics. We cannot assume that architecture's purpose can be described simply from a scientific or mathematical perspective; the enduring role of architecture in society is often linked to its material presence, its historic significance, or its capacity to represent a set of otherwise-intangible values (Ostwald and Vaughan 2016: 21). In fact, architecture has many aspects, among which, is that we should value the material and spiritual needs of humans and pave the way for their evolution. Form and geometry might be the only means to reach the goal. As a result, the best lesson that nature can give us is to find our appropriate place in nature as an important principle. Furthermore, we should learn the reason behind the natural forms in nature while negligence results in failure to achieve the desired architecture goals.

\section{References}

Betsky, A. (1990). Violated Perfection: Architecture and the Fragmentation of the Modern. Rizzoli, New York.

Bovill, C. (1996). Fractal Geometry in Architecture and Design. Birkhäuser, Boston.

Burckhardt, T. (2003). Principes et méthodes de l'art sacré, (Trans. J. Sattari), Soroush Publications, Tehran.

Eisenman, P. (1988). “Eisenmanesie”. V: Architecture + Urbanism, Vol. Extra edition. August. p.:70.

Harris, J. (2007). Integrated function systems and organic architecture from Wright to Mondrian. Nexus Network Journal, 9(1), pp. 93-101.
Jencks, C. (2004). The architecture of the jumping universe, (Trans. V. Ghobadian \& D. Sattarzadeh), Tabriz Islamic Azad University Publications, Tabriz.

Joy, Y. (2011). A review of the presence and use of fractal geometry in architectural design. Environment and Planning B: Planning and Design Journal, 38(5), pp. 814-828.

Langhof, C. (1994). Imagination is More Important than Knowledge. Curtin University, Architecture Document.

Lorentz, E. (1963). Deterministic nonperiodic flow. Journal of Atmospheric Sciences, 20, pp. 130-141.

Lorenz, W . E. (2003). Fractals and fractal architecture. Master's thesis, Vienna University of Technology.

Mandelbrot, B. (1982). The Fractal Geometry of Nature. W. H. Freeman and Company, New York.

Mandelbrot, B. (1992). Fractal geometry: A descriptor of nature, (Trans: M. Bagheri), Daneshmand Journal, 29(338), pp. 35-42.

Ostwald, M. J. (2001). Fractal architecture: Late twentieth century connections between architecture and fractal geometry. Nexus Network Journal, 3(1), pp. 73-83.

Ostwald, M. J. (2010). The politics of fractal geometry in Russian paper architecture: The intelligent market and the cube of infinity. Architectural Theory Review, 15(2), pp. 125-137.

Ostwald, M. J., \& Williams, K. (2015). Mathematics in, of and for architecture: A framework of types. In: Williams, K., \& Ostwald, M. (eds.), Architecture and Mathematics from Antiquity to the Future. Springer International Publishing, Switzerland, pp. 31-57.

Ostwald, M. J., \& Vaughan, J. (2016). The fractal dimension of architecture. In: Williams, K. (ed.), Mathematics and the Built Environment. Springer International Publishing, Switzerland, pp. 21-37.

Pawley, M. (1994). Fall off your chair at the folly. The Observer, May 22.

Pearson, D. (2001). New Organic Architecture, the Breaking Waves. University of California Press, California.

Prigogine, I. (1984). Order Out of Chaos, the Evolutionary Paradigm and the Physical Sciences. Bantam Books, New York.

Salingaros, N. (2004). Fractals in the new architecture, (Trans: N. Chitsazan \& N. Iranmanesh), Architect Journal, 26, pp. 26-29.

Sardar, Z. (2001). Introducing Chaos, (Trans: A. Gharib). Shirazeh Publications, Tehran.

Shepheard, P. (1994). What is Architecture: An Essay on Landscapes, Buildings, and Machines. MIT Press, Cambridge \& Massachusetts.

Sorkin, M. (1991). Nineteen millennial mantras. In: Noever P (ed.), Architecture in Transition: Between Deconstruction and New Modernism. Prestel, Munich.

Vaughan, J., \& Ostwald, M. J. (2017). The comparative numerical analysis of nature and architecture: A new framework. International Journal of Design \& Nature and Ecodynamics, 12(2), pp. 156-166. 